\title{
Investigation of a Two-Dimensional Hot Jet's Infrared Radiation Using Four Different Turbulence Models
}

\author{
Wei Huang and Hong-hu Ji \\ Jiangsu Province Key Laboratory of Aerospace Power System, Nanjing University of Aeronautics and Astronautics, \\ Nanjing 210016, China \\ Correspondence should be addressed to Wei Huang; hw_one@163.com
}

Received 11 March 2014; Revised 23 July 2014; Accepted 23 July 2014; Published 28 September 2014

Academic Editor: Stefan Balint

Copyright (C) 2014 W. Huang and H.-h. Ji. This is an open access article distributed under the Creative Commons Attribution License, which permits unrestricted use, distribution, and reproduction in any medium, provided the original work is properly cited.

\begin{abstract}
A two-dimensional (2D) jet flow and temperature field are simulated by using $k-\varepsilon$ T. C. model and compared with other three nontemperature corrected models, which are standard $k-\varepsilon$, RNG $k-\varepsilon$, and SST $k-\omega$ model. Then based on the calculated results, the spectral infrared radiation characteristics within $4 \sim 5 \mu \mathrm{m}$ of the $2 \mathrm{D}$ jet flow were calculated. By comparing the computed results of the velocity, temperature field, and infrared radiation with the experimental measurements, it shows that the $k-\varepsilon$ T. C. model predicts mean flow mixing more rapidly and the turbulent kinetic energy dissipates earlier than with no temperature correction; the $k-\varepsilon$ T. C. model could give a good prediction for the velocity and temperature distributions on the centerline of the $2 \mathrm{D}$ hot gas jet, but not on the locations off the centerline. The maximum computation error of the 2D hot jet infrared radiation is decreased from $86 \%$ to $26 \%$, and the accuracy of the computation is greatly improved.
\end{abstract}

\section{Introduction}

The effect of infrared radiation of hot jets is of major importance in the target detection and recognition. Twodimensional (2D) nozzles which can produce a strong secondary flow are usually applied to reduce the infrared signature of the hot jets [1-3]. Therefore the improvement of the computation accuracy of $2 \mathrm{D}$ hot jet's infrared radiation is of great significance.

The computation of infrared radiation of hot jets relies on accurate simulation of temperature field. Currently, the most commonly used technique to compute the temperature field is to solve the Reynolds Averaged Navier-Stokes (RANS) equations [4] and scalar conservation equations. These equations are solved with turbulence models, which establish the relation between the Reynolds stress in RANS equations and the turbulent scalar flux in Reynolds averaged scalar equations with the velocity gradient and scalar (such as temperature and concentration) gradient of the flow. Therefore, the turbulence model adopted in the calculation will affect the accuracy of the hot jet temperature filed and in turn affect the calculation accuracy of the infrared radiation.

There are dozens of turbulence models, of them twoequation models, such as standard $k-\varepsilon$ model [5], RNG $k-\varepsilon$ model [6], and SST $k-\omega$ model [7], are currently widely used in engineering [8]. Because the parameters in the equations of these models are calibrated with simple shear flow at the condition of room temperature and low Mach number without considering the variation of temperature, they are often difficult to accurately simulate high temperature jet flow. For example, Dembowski and Georgiadis [9] numericaly simulated the velocity and temperature field of a nozzle with SST $k-\omega$ model and Chien $k-\varepsilon$ model and compared with Seiner's et al. [10] experiment data, the results show that accurate results can only be obtained when the temperature of jet is not high, for example, between $313 \mathrm{~K}$ and $508 \mathrm{~K}$, and then the deviation will significantly increase while the temperature increases from $508 \mathrm{~K}$ to $1366 \mathrm{~K}$.

In order to make the two-equation turbulence models still be applicable to the high temperature jets, Abdol-Hamid et al. 
[11] proposed a $k-\varepsilon$ model based on temperature correction, namely, $k-\varepsilon$ T. C. model, in which the eddy viscosity in the model is modified by the local total temperature gradient normalized by the local turbulence length scale. Results show that the model has a good adaptability to both subsonic and supersonic jets with a wide temperature range. Tam and Ganesan [12] proposed a modified $k-\varepsilon$ turbulence model to include the effect of the presence of a large-density gradient. In addition, Massey et al. [13] also used $k-\varepsilon$ T. C. to study the separation flow of chevron nozzle and got the similar results to Abdol-Hamid. Georgiadis et al. [14] evaluated the $k-\varepsilon$ T. C. model (or PAB T. C.) for round jet flow; the results showed that the modified model improved predictions of mean axial velocities for the heated jet but did not improve prediction of the calculated turbulent kinetic energy fields. The study of Li et al. $[15,16]$ showed that those modified $k$ - $\varepsilon$ model provided improved infrared radiation predictions for the same round jet.

While there are considerable experimental and computational studies [17-19] on cold jet flow of two-dimensional (rectangular) nozzle, there are comparatively few studies on hot jet flow of this type of nozzle, especially the infrared radiation. The main purpose of this paper is to study how the two-equation turbulence models influence the temperature and infrared radiation of a $2 \mathrm{D}$ jet flow. The turbulence models studied includes standard $k-\varepsilon$, RNG $k-\varepsilon$, SST $k-\omega$, and $k$ $\varepsilon$ T. C. models. By comparing the computed results of the velocity, temperature field, and infrared radiation with the experimental measurements, it shows that the calculation with $k-\varepsilon$ T. C. model greatly improved the accuracy of the computation.

\section{Computation Method of Hot Jet's Infrared}

2.1. Procedure of Hot Jet Infrared Radiation Computation. The essence of hot jet's infrared computation is solving the radiation transfer equation, which depends on the temperature and species concentration distributions of the jet flow. The temperature and species concentration distributions were obtained by solving the momentum, energy, and species transfer equations by the method of CFD.

The procedure of hot jet infrared radiation computation is a complex process, which involves nozzle geometry modeling, CFD computation domain meshing, CFD computation, IR (infrared radiation) computation, correction of atmosphere transmittance, and results display. The relationship between CFD computation and infrared radiation computation is shown in Figure 1. As shown in the figure, the computation was divided into two modules, which are commercial CFD module and IR module, respectively. The temperature, pressure, and species concentration of hot jet outputted from CFD module were interpolated into the IR module. The results of CFD module were greatly affected by the turbulence model, and four different turbulence models will be discussed in the next section.

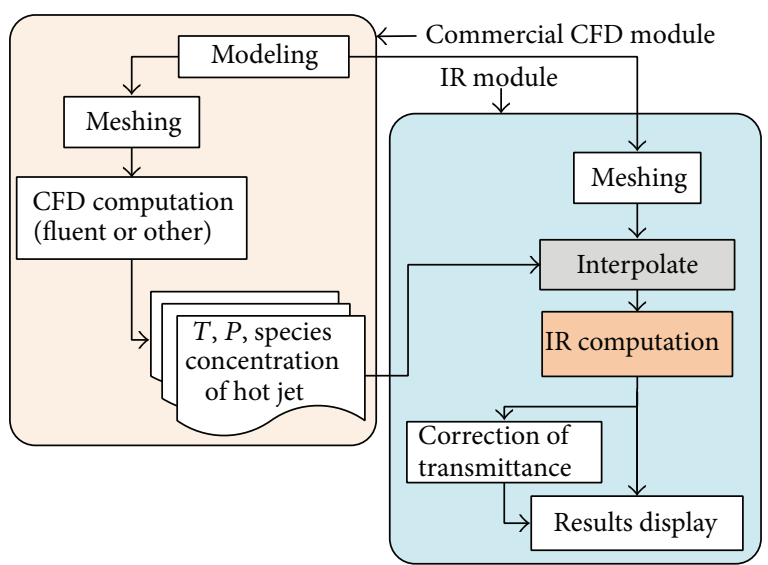

FIGURE 1: The relationship between CFD computation and infrared radiation computation.

2.2. Four Two-Equation Turbulence Models. In the scheme of eddy viscosity models (EVM), the Reynolds stress $-\rho \overline{u_{i} u_{j}}$ and turbulent scalar flux $\rho \overline{u_{i} \theta}$ are determined with

$$
\begin{gathered}
-\rho \overline{u_{i} u_{j}}=\mu_{t}\left[\frac{\partial U_{i}}{\partial x_{j}}+\frac{\partial U_{j}}{\partial x_{i}}\right]-\frac{2}{3} \rho k \delta_{i j}, \\
\rho \overline{u_{i} \theta}=-\frac{\mu_{t}}{\sigma_{\theta}} \frac{\partial \Theta}{\partial x_{i}}
\end{gathered}
$$

where $U_{i}$ and $u_{i}$ are mean and fluctuation velocity in $i$ direction, respectively, $\Theta$ and $\theta$ are mean and fluctuation scalar, respectively, which could be temperature or species concentration, $k$ is turbulent kinetic energy, and $\sigma_{\theta}$ is turbulent Prandtl number or turbulent Schmidt number. $\mu_{t}$ is eddy viscosity, which is determined with different turbulent models. The Prandtl number takes 0.85, and the Schmidt number takes 0.7 in this paper.

2.2.1. Standard $k-\varepsilon$ Model. In standard $k-\varepsilon$ model [5], the expression for $\mu_{t}$ is defined by

$$
\mu_{t}=\rho C_{\mu} \frac{k^{2}}{\varepsilon}
$$

where turbulent kinetic energy $k$ and its dissipation rate $\varepsilon$ are determined by solving their transport equations as follows:

$$
\begin{aligned}
& \frac{\partial}{\partial t}(\rho k)+\frac{\partial}{\partial x_{i}}\left(\rho k U_{i}\right) \\
& \quad=\frac{\partial}{\partial x_{j}}\left[\left(\mu+\frac{\mu_{t}}{\sigma_{k}}\right) \frac{\partial k}{\partial x_{j}}\right]-\rho \overline{u_{i} u_{j}} \frac{\partial U_{j}}{\partial x_{i}}-\rho \varepsilon
\end{aligned}
$$




$$
\begin{aligned}
\frac{\partial}{\partial t} & (\rho \varepsilon)+\frac{\partial}{\partial x_{i}}\left(\rho \varepsilon U_{i}\right) \\
& =\frac{\partial}{\partial x_{j}}\left[\left(\mu+\frac{\mu_{t}}{\sigma_{\varepsilon}}\right) \frac{\partial \varepsilon}{\partial x_{j}}\right]-\rho \overline{u_{i} u_{j}} \cdot C_{1 \varepsilon} \frac{\varepsilon}{k}-C_{2 \varepsilon} \varepsilon \frac{\varepsilon^{2}}{k},
\end{aligned}
$$

where $C_{\mu}=0.09, \sigma_{k}=1.0, \sigma_{\varepsilon}=1.3, C_{1 \varepsilon}=1.44$, and $C_{2 \varepsilon}=$ 1.92 .

2.2.2. RNG $k$ - $\varepsilon$ Model. The RNG $k-\varepsilon$ model [6] was derived using a statistical technique called renormalization group theory. It is similar in form to the standard $k-\varepsilon$ model, but it includes the following refinements: (1) the RNG model has an additional term in its $\varepsilon$ equation that improves the accuracy for rapidly strained flows; (2) the effect of swirl on turbulence is included in the RNG $k-\varepsilon$ model in order to improve the accuracy for swirling flows; (3) the renormalization group theory provides an analytical formula for turbulent Prandtl number, while the standard $k-\varepsilon$ model only uses constant values. With these features, the RNG $k-\varepsilon$ model becomes more accurate and reliable for wider class of flows than the standard $k-\varepsilon$ model. In RNG model, $C_{\mu}=0.085, C_{1 \varepsilon}=1.42$, and $C_{2 \varepsilon}=1.68, \sigma_{k}=\sigma_{\varepsilon}=0.7179$.

2.2.3. SST $k-\omega$ Model. The Shear-Stress Transport (SST) $k-\omega$ model was developed by Menter [7] to effectively combine the robust and accurate formulation of the $k-\omega$ model in the near-wall region with the free stream independence of the $k-\varepsilon$ model in the distanced field. The definition of eddy viscosity is

$$
\mu_{t}=\frac{\rho a_{1} k}{\max \left(a_{1} \omega / \alpha^{*}, S F_{2}\right)},
$$

where $a_{1}=0.031$. The function of $\omega$ is defined as

$$
\omega=\frac{\varepsilon}{\left(\beta^{*} k\right)},
$$

with $\beta^{*}=0.09 . S$ is strain rate magnitude; $F_{2}=\tanh \left(\Phi_{2}^{4}\right)$. The functions of $\Phi_{2}$ are

$$
\Phi_{2}=\max \left[2 \frac{\sqrt{k}}{0.09 \omega y}, \frac{500 \mu}{\rho y^{2} \omega}\right] .
$$

The transport equations of the SST $k$ - $\omega$ model are

$$
\begin{aligned}
& \frac{\partial}{\partial t}(\rho k)+\frac{\partial}{\partial x_{i}}\left(\rho k U_{i}\right) \\
& =\frac{\partial}{\partial x_{j}}\left[\left(\mu+\frac{\mu_{t}}{\sigma_{k}}\right) \frac{\partial k}{\partial x_{j}}\right]+\widetilde{G}_{k}-\rho \beta^{*} k \omega, \\
& \frac{\partial}{\partial t}(\rho \omega)+\frac{\partial}{\partial x_{i}}\left(\rho \omega U_{i}\right) \\
& =\frac{\partial}{\partial x_{j}}\left[\left(\mu+\frac{\mu_{t}}{\sigma_{\omega}}\right) \frac{\partial \omega}{\partial x_{j}}\right]+\frac{\rho \alpha}{\mu_{t}} \widetilde{G}_{k}-\rho \beta \omega^{2} .
\end{aligned}
$$

In these equations, $\widetilde{G}_{k}$ represents the generation of turbulence kinetic energy due to mean velocity gradients. The $\widetilde{G}_{k}$ term is defined as

$$
\widetilde{G}_{k}=\min \left(\mu_{t} S^{2}, 10 \rho \beta^{*} k \omega\right) .
$$

The more detailed description of this model could be found in $[20]$.

2.2.4. $k-\varepsilon$ T. C. Model. The $k-\varepsilon$ T. C. model [12] has the same transport equations (as shown in (3)) as standard $k-\varepsilon$ model, but the eddy viscosity is corrected by the local total temperature gradient as follows:

$$
\mu_{t}=\rho C_{\mu} C_{T} \frac{k^{2}}{\varepsilon}
$$

The correction coefficient, $C_{T}$, is calculated with (8) as follows:

$$
C_{T}=1+\frac{\bar{T}^{3}}{0.041+f\left(M_{t}\right)},
$$

where

$$
\bar{T}=\frac{k^{3 / 2}}{\varepsilon} \frac{\left\|\nabla T_{t}\right\|}{T_{t}}
$$

$T_{t}$ is stagnation temperature. The $f\left(M_{t}\right)$ function is a correction factor to improve the applicability of the model to highspeed flow; $f\left(M_{t}\right)$ is defined as

$$
f\left(M_{t}\right)=\left(M_{t}^{2}-M_{t 0}^{2}\right) H\left(M_{t}-M_{t 0}\right),
$$

where $M_{t}=\sqrt{2 k} / a, M_{t 0}=0.1, a$ is local acoustic speed, and $H(x)$ is Heaviside step function.

\subsection{The Method to Solve Radiation Transport Equation.} Infrared radiation transmission in any direction of $\vec{S}$ is governed by the radiation transport equation [20] (14). Consider

$$
\frac{d L_{\lambda}(s, \vec{S})}{d s}=-\kappa_{\lambda}(s) L_{\lambda}(s, \vec{S})+\frac{\kappa_{\lambda}(s)}{\pi} E_{b \lambda}(T(s)),
$$

where $L_{\lambda}$ is spectral radiance, $s$ is the local location, $\kappa_{\lambda}$ is spectral absorption coefficient, $E_{b \lambda}$ is blackbody emissive power, and $T$ is local temperature.

By integrating (14) along the ray, the total radiance of the hot jets along the direction of $\vec{S}$ can be obtained. The ray tracing method is used to calculate the infrared radiation transmission of the hot jet. The illustration of the ray tracing method is shown in Figure 2.

The computational expression of $L_{\lambda}(\vec{S})$ is

$$
\begin{gathered}
L_{\lambda}(\vec{S})=\sum_{k=1}^{N}\left[1-e^{-\kappa_{\lambda}(k) \delta s}\right] \frac{E_{b \lambda}(T(k))}{\pi} \\
\cdot \prod_{i=1}^{k-1} e^{-\kappa_{\lambda}(i) \delta s}, \quad(k \geq 2), \\
L_{\lambda}(\vec{S})=\left[1-e^{-\kappa_{\lambda}(k) \delta s}\right] \frac{E_{b \lambda}(T(k))}{\pi}, \quad(k=1) .
\end{gathered}
$$




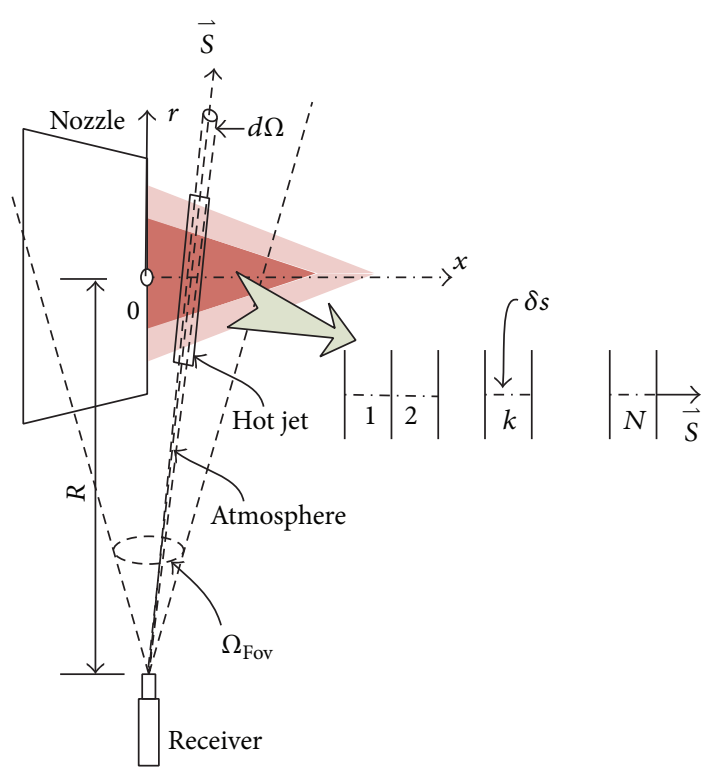

FIGURE 2: The illustration of the ray tracing method.

In this work, the narrow band model is used to calculate the absorption coefficient $\kappa_{\lambda}$ and the spectral step is $10 \mathrm{~cm}^{-1}$. The original data of $\kappa_{\lambda}$ can be found in NASA SP-3080 or HITEMP database.

The transmittance of the air between the target and the receiver at the band of $4.26 \mu \mathrm{m}$ is necessary to consider, although the concentration of carbon dioxide in the air is very less. So the total radiation intensity in the field of view $\Omega_{\text {FOV }}$ (see Figure 2) between wavelength $\lambda_{1}$ and $\lambda_{2}$ can be calculated by using

$$
I_{\lambda_{1}-\lambda_{2}}=\int_{\lambda_{1}}^{\lambda_{2}} \int_{\Omega_{\mathrm{FOV}}} L_{\lambda}(\vec{S}) R^{2} \tau_{\lambda}^{\text {atmosphere }} d \Omega d \lambda,
$$

where $R$ is the distance between the hot jets and the receiver. $\tau_{\lambda}^{\text {atmosphere }}$ is the transmissivity of atmosphere between the hot jets and the receiver, and it was calculated by using software Modtran. In the calculation, the atmosphere is treated as a medium without scattering. The composition of the midlatitude summer atmosphere is used to compute atmosphere attenuation.

From the above equations, we can see that the infrared radiation or radiant intensity is mainly dependent on both the temperature and the absorption coefficient of the hot jets.

\section{Experimental Measurement of Velocity, Temperature, and Infrared Radiation}

3.1. Test Model and Measurement Arrangement. A 2D nozzle with aspect ratio of 6 is used in present study, as shown in Figure 3, the corss section of which is gradually transformed from circular to rectangular. The equivalent diameter of the nozzle outlet is defined as $D_{e}=4 A / C$, where $A$ is area and $C$ is perimeter. The mixer divides the axisymmetric part of the nozzle into two channels, the main channel and bypass channel. The main gas stream has a total temperature of

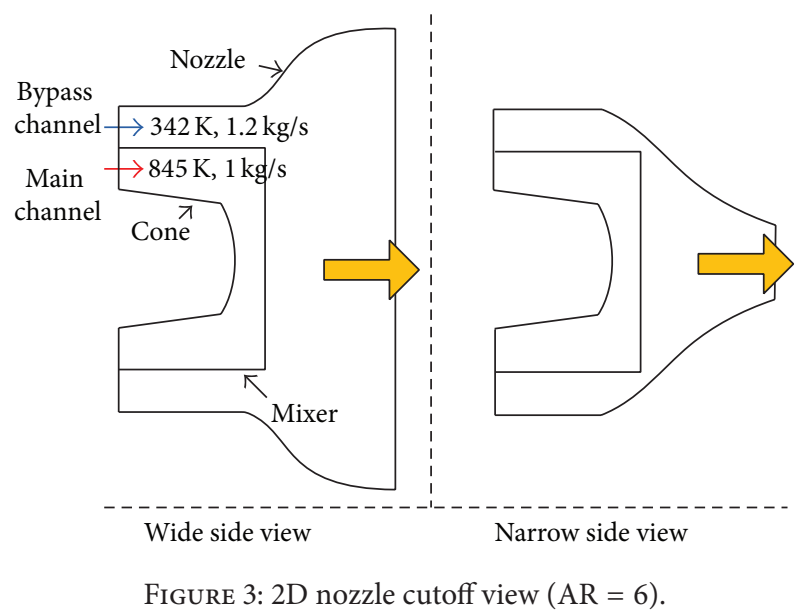

$845 \mathrm{~K}$ and mass flow rate of $1 \mathrm{~kg} / \mathrm{s}$. The bypass airflow has a total temperature of $342 \mathrm{~K}$ and mass flow rate of $1.2 \mathrm{~kg} / \mathrm{s}$. The Mach number of nozzle outlet is about 0.15 , and the Reynolds number is about $7.1 \times 10^{5}$.

The measurement scenario of pressure, temperature, and infrared radiation of the gas jet is shown in Figures 4(a) and 4(b). The temperature distribution was measured by thermocouples rake, and the total pressure and the static pressure are measured by the pitot tubes rake. The measurement was made within the range from the nozzle exit to $6 D_{e}$ downstream. Based on the experiments, the velocity of the gas flow can be calculated. The infrared radiation of the gas jets on the direction of $90^{\circ}$ to the axis of the nozzle is measured using a FT-spectroradiometer which has a wavelength band $4 \sim 5 \mu \mathrm{m}$ and located 34 meters away from the nozzle. The measurements were made in both wide and narrow sides of the 2D nozzle, as shown in Figure 4(b).

3.2. Measurement Results. The measurement results include centerline velocity, temperature, radial velocity, temperature, and spectral infrared radiation, which are given in Figures 10, 14 , and 15 . The detailed description is omitted here.

3.3. Measurement Uncertainty Analysis. The temperature measurement accuracy with thermocouples is influenced by shunting error, response time error, and radiation error. The type of the thermocouple was commercial $K$-type sheathed thermocouple. A multichannel temperature inspection instrument was used to acquire the temperature values. Considering the uncertainty of temperature inspection instrument, the total uncertainty of temperature measurement is about $\pm\left(1 \% \mathrm{~T}+1.0^{\circ} \mathrm{C}\right)$.

The uncertainties of velocity measurement include the uncertainty of both temperature and pressure. The angles between the pitots and the jet axial component were less than $10^{\circ}$; the total uncertainty is about $\pm 2 \%$.

The infrared radiation measurement accuracy with FTspectroradiometer is influenced by three types of errors. The first type of errors is calibration source errors, which include the error of blackbody temperature, about $2.5 \%$, and the error of blackbody emissivity over the operational spectral 


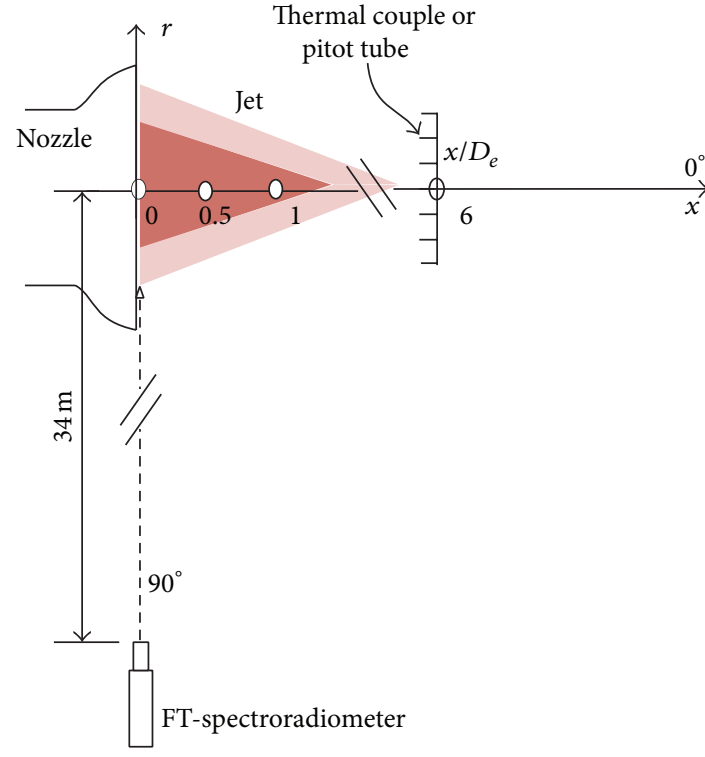

(a) The instrumentation of the measurement

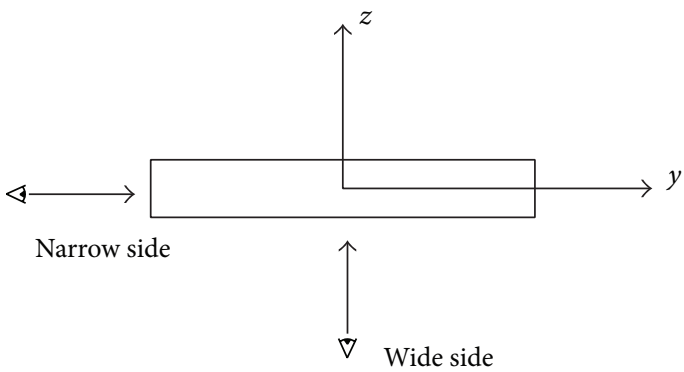

(b) The sketch map of narrow side measurement and wide side measurement

FIGURE 4: The measurement scenario of temperature, pressure, and infrared radiation.

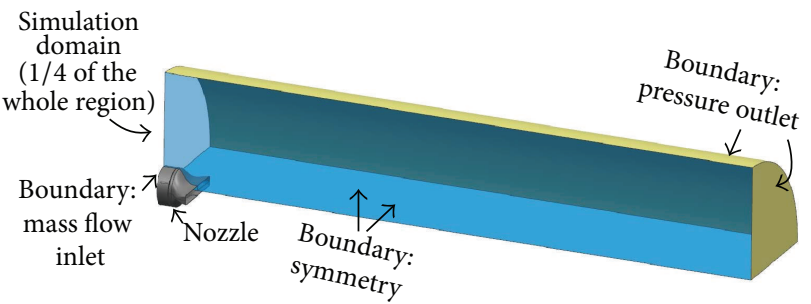

FIGURE 5: Simulation domain and boundary types.

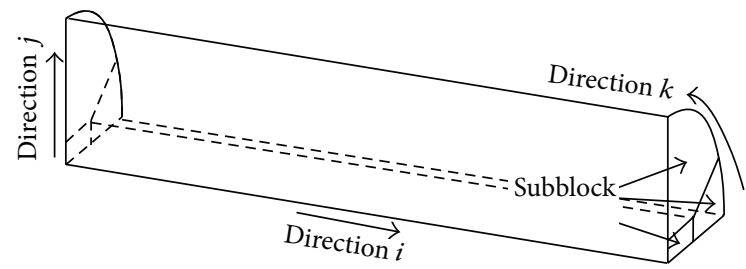

FIGURE 6: Schematic diagram of meshing method.

range, about $2 \%$. The second type of errors is calibration drift, which is about $2 \%$. Finally, the third type of errors is the spectrometer intrinsic linearity, which is about $1 \%$. These errors are independent of each other, and the total error is calculated by adding up three errors' contribution in rootsum-square fashion, which is about $4 \%$.

\section{Verification of the CFD and IR Computation}

The distributions of flow field, temperature field, and species concentration field are computed using the CFD software
Fluent [21]. For CFD code, the most important numerical errors and uncertainties are due to use of iterative solution methods and specification of various input parameters such as spatial and time step sizes and other parameters [22]. Since the steady-state simulations are carried out, iteration number and grids size are the major contributions to the uncertainties [23].

4.1. Calculation Domain, Boundary Condition, and Grids Generation. Since the nozzle is symmetrical, in order to save computing time, only $1 / 4$ of the whole region was simulated. The simulation domain and boundary types are shown in Figure 5. The boundary condition of main channel and bypass channel is set to mass flow inlet, and the boundary condition of simulation domain is set to pressure outlet. The boundary condition of symmetric plane is set to symmetry.

The nozzle geometry was complex, and in order to get structured grid, the O-grid method was applied to the meshing domain. The schematic diagram of meshing method is shown in Figure 6. Fan-shaped region is divided into several subblocks. The block has three directions, which are $i, j$, and $k$.

Uncertainties due to grid size are estimated using systematically refined grids with constant refinement ration, $r_{G}=\sqrt{2}$. Grid studies were conducted using three grids. Grid dimensions for grid refinement studies are given in Table 1, and a comparison of the three grids at symmetry plane is shown in Figure 7. The total number of cells of grid 2 and 3 is about 2.7 and 2.9 times to that of gird 1 and 2 , respectively. The product of $i \times j \times k$ does not equal the total number of cells and this is due to the way of meshing. In all cases, the simulation domain extended roughly $22 D_{e}$ downstream of the nozzle exit and $4.2 D_{e}$ from the centerline in the radial direction. 


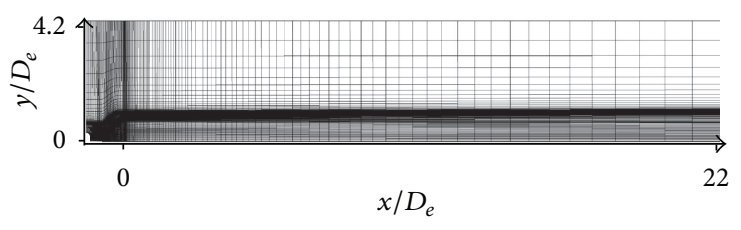

(a) Coarsest-grid 1

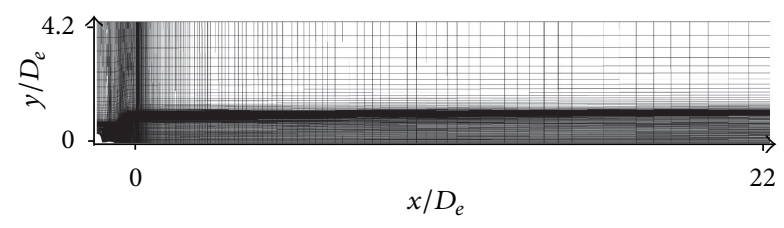

(b) Medium-grid 2

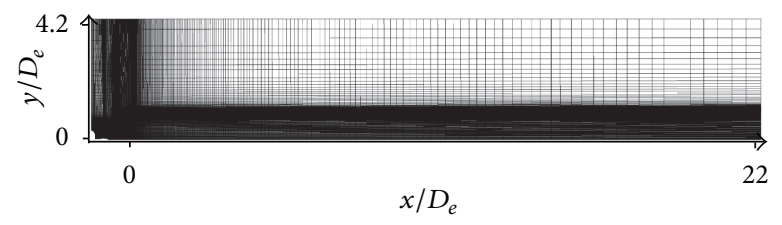

(c) Finest-grid 3

FIGURE 7: Grids of symmetry plane from verification studies for 2D hot jet.

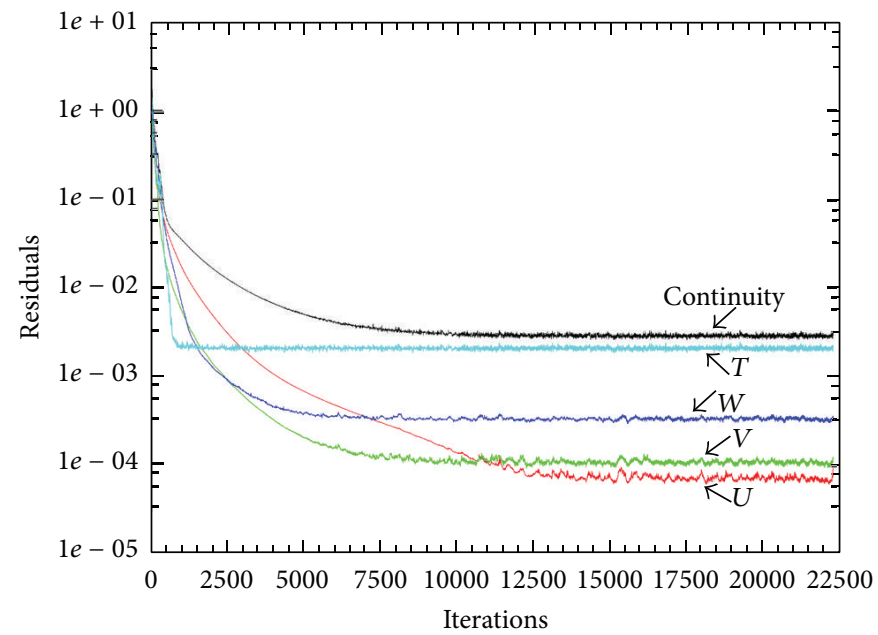

(a) Solution residuals change

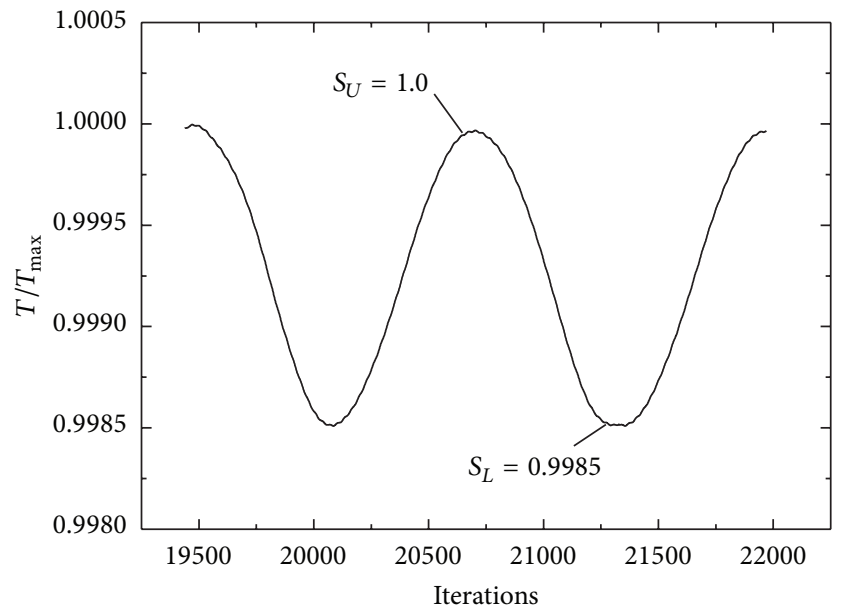

(b) Dimensional temperature at nozzle outlet

FIGURE 8: Iteration history for 2D nozzle with finest-gird 3.

4.2. Numerical Solution of RANS CFD Code. The governing equations described above were solved numerically using commercial software, Fluent. Standard $k-\varepsilon$ model, RNG $k$ $\varepsilon$ model, and SST $k-\omega$ model can be directly selected in the software. The $k-\varepsilon$ T. C. model was achieved by embedding a UDF function in the software. The density is calculated using the ideal gas model, and the molecule viscosity is calculated using the Sutherland model. An implicit solution based on the finite volume method (FVM) along with a quadrilateral nonuniform structured grid in the Cartesian coordinate system was used. The flow, turbulent kinetic energy, and specific dissipation rate equations were discretized using the second-order upwind scheme at the cell faces.

\subsection{Numerical Uncertainties Analysis of CFD Computation.} The convergence criterion of numerical computation is that the solution residuals and nozzle outlet temperature do not change significantly with the number of iterations. Iteration history for 2D nozzle on gird 3 is shown in Figure 8. As shown in the figure, the solution changes drop three to five orders of magnitude. The variation in dimensional temperature at nozzle outlet is about $0.15 \% S_{3}$ (where $S_{3}$ is the solution on the finest-grid 3) over the last period of oscillation. Iterative uncertainty is estimated as half of the range of the maximum and minimum values over the last two periods of oscillation, so the iterative uncertainty is about $0.075 \%$.

Grid convergence is assessed through core lengths solutions on three systematically refined grids with constant refinement ratio (see Table 1). The core length is defined for the $x / D_{e}$ in which $T=0.995 T_{e}$ (where $T_{e}$ is the temperature at the nozzle exit) along the axis of the jet. Simulated core lengths using $k-\varepsilon$ T. C. and RNG $k-\varepsilon$ model are shown in Table 2 . The core lengths using $k-\varepsilon$ T. C. model are much shorter than RNG $k-\varepsilon$ model and display monotonic convergence with the increase in the number of gird cells. According to Stern and Wilson theory [23], the estimated grid uncertainty is less than $0.82 \%$ and $1.4 \%$ for $k-\varepsilon$ T. C. model and 


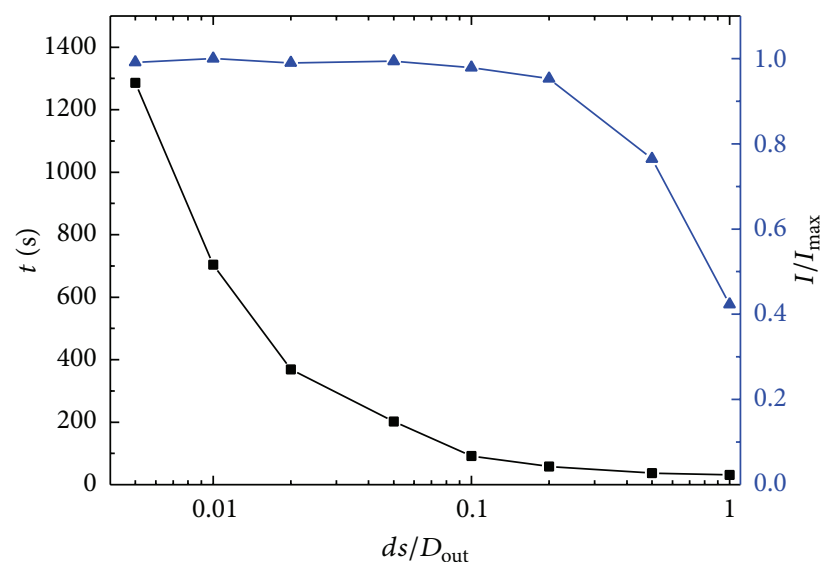

$\rightarrow$ Consumed time

$\rightarrow$ Intensity

FIGURE 9: Influence of ray discrete step $d s$ on radiation intensity and consumed time.

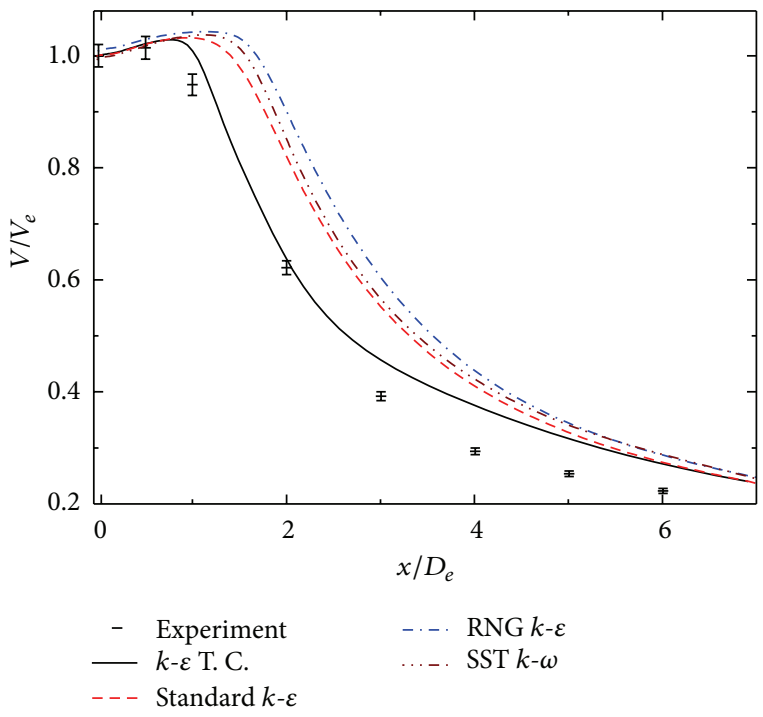

FIGURE 10: Computed centerline velocity of the jet compared to experiment data.

TABLE 1: Grid dimensions for grid refinement.

\begin{tabular}{lcc}
\hline Grid & Grid dimensions $(i \times j \times k)$ & Total number of cells \\
\hline 1 & $160 \times 100 \times 50$ & 526,150 \\
2 & $226 \times 140 \times 70$ & $1,406,352$ \\
3 & $320 \times 200 \times 100$ & $4,078,570$ \\
\hline
\end{tabular}

RNG $k-\varepsilon$ model, respectively. The core lengths using standard $k-\varepsilon$ and SST $k-\omega$ modelare between $k-\varepsilon$ T. C. and RNG $k-$ $\varepsilon$ model, and the uncertainties of these two models may be between $0.82 \%$ and $1.4 \%$.

The difference of core length with different turbulence models exceeds $100 \%$, which is obviously greater than the numerical uncertainty using the finest-grid 3 . The following computation and analysis were all carried out on grid 3.

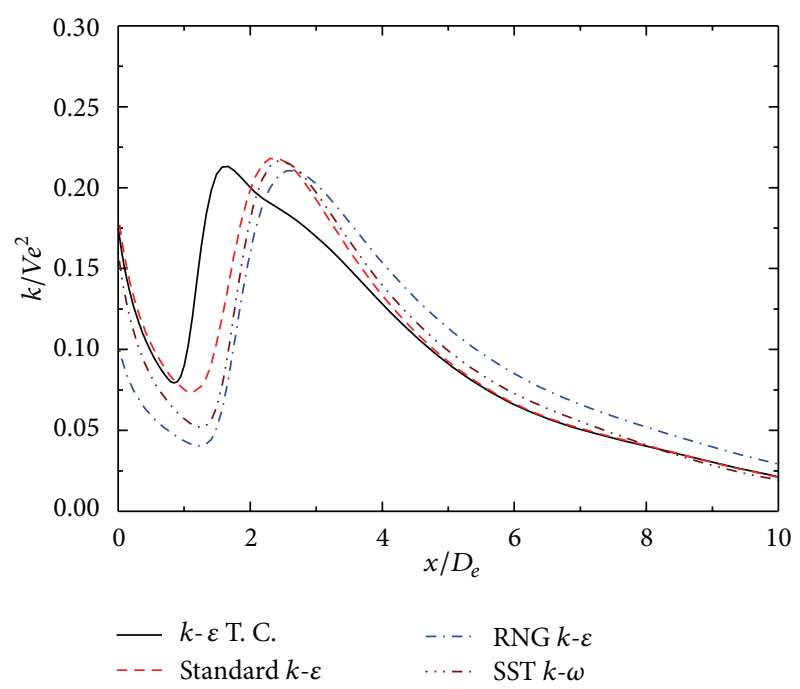

FIGURE 11: Computed centerline turbulent kinetic energy profiles of the jet.

4.4. Numerical Uncertainties Analysis of IR Computation. An important factor that affects the IR computation is the size of ray discrete step $d s$ (see Figure 2). The influence of ray discrete step $d s$ on radiation intensity and consumed time is shown in Figure 9. The shorter the $d s / D_{e}$, the more stable the radiation intensity, and the more time spent. The numerical uncertainties of IR computation are about $0.5 \%$ when $d s / D_{e}<0.05$. In the following calculations, $d s / D_{e}$ were taken as 0.05 .

\section{Results and Analysis}

5.1. Velocity and Turbulent Kinetic Energy Distribution of the Jet. A comparison of centerline axial velocities profiles obtained from the four turbulence models is made with experimental data for the $2 \mathrm{D}$ jet in Figure 10. It shows that the RNG $k-\varepsilon$ model gives the longest length of potential core region which is approximately 1.8 times longer than the measurement values. Beyond the potential core, however, the rate of jet decay is slower than that produced by the other models and the experimental data. The standard $k-\varepsilon$ and SST $k-\omega$ models get similar predicted length of the core region that is about 1.2 times longer and then downstream mixing rates that are also too slow relative to the experimental data. The $k-\varepsilon$ T. C. model dramatically improves the accuracy of the predicted length of the velocity core region because the correction of eddy viscosity is only 0.2 times longer than the measured data. The $k-\varepsilon$ T. C. model results have a slightly slow rate of jet decay compared to experimental data.

The computed centerline turbulent kinetic energy profiles and the turbulent kinetic energy contours of the two symmetric planes are compared in Figure 11 to Figure 13. The decline change which appears between $x / D_{e}=0 \sim 1$ is caused by reflow phenomenon after central cone. The RNG $k-\varepsilon$ model results show the slowest propagation of turbulence to the jet centerline. The standard $k-\varepsilon$ and SST $k-\omega$ models approaches 
TABLE 2: Core lengths and numerical uncertainty of systematically refined grids.

\begin{tabular}{|c|c|c|c|c|}
\hline \multirow{2}{*}{ Grid } & \multicolumn{2}{|c|}{$k-\varepsilon$ T. C. model } & \multicolumn{2}{|c|}{ RNG $k-\varepsilon$ model } \\
\hline & Core length $\left(D_{e}\right)$ & Uncertainty (finest-grid 3) & Core length $\left(D_{e}\right)$ & Uncertainty (finest-grid 3) \\
\hline 1 & 0.568 & - & 1.131 & - \\
\hline 2 & 0.586 & - & 1.199 & - \\
\hline 3 & 0.593 & $<0.82 \%$ & 1.221 & $<1.4 \%$ \\
\hline
\end{tabular}

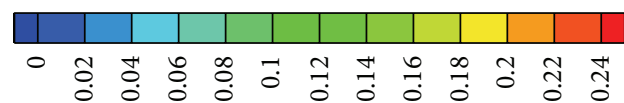

$k / V e^{2}$

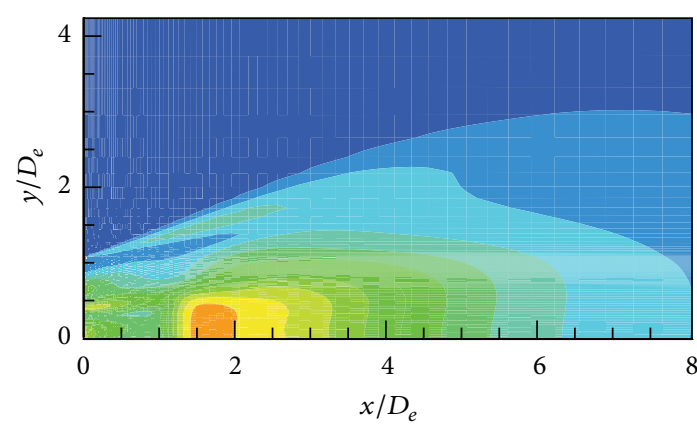

(a) $k-\varepsilon$ T. C.

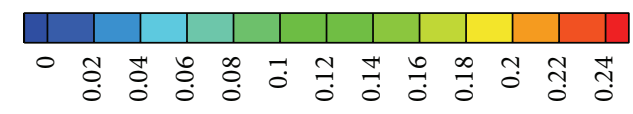

$k / V e^{2}$

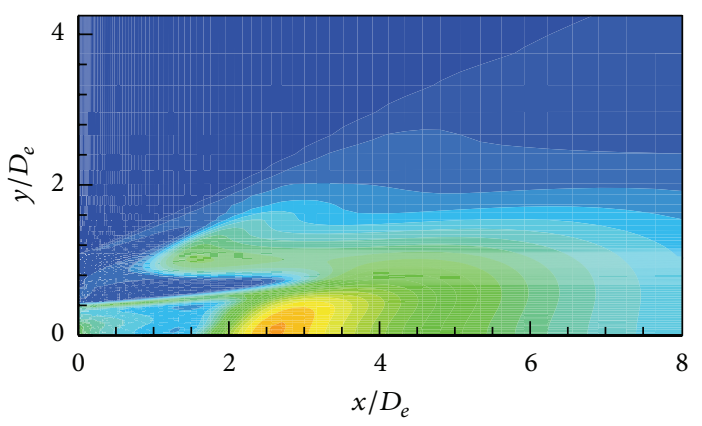

(c) RNG $k-\varepsilon$
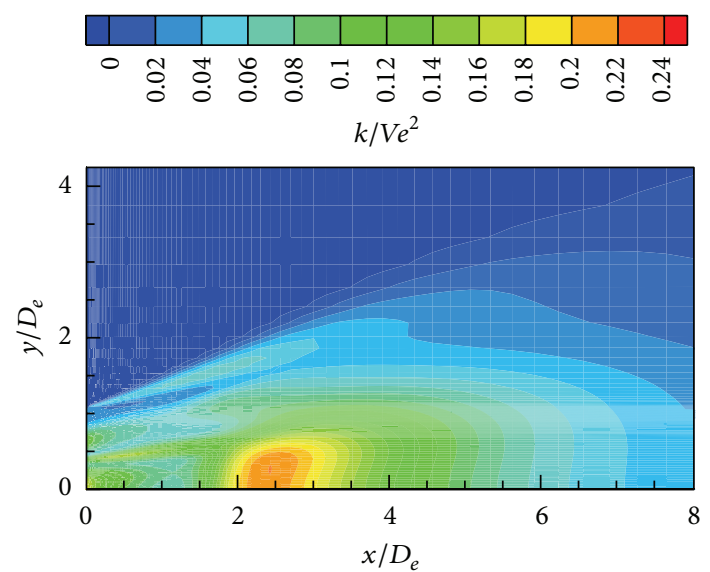

(b) Standard $k-\varepsilon$

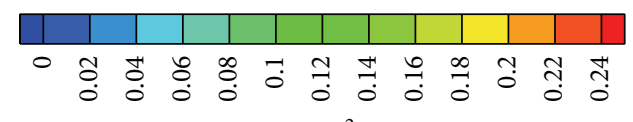

$k / V e^{2}$

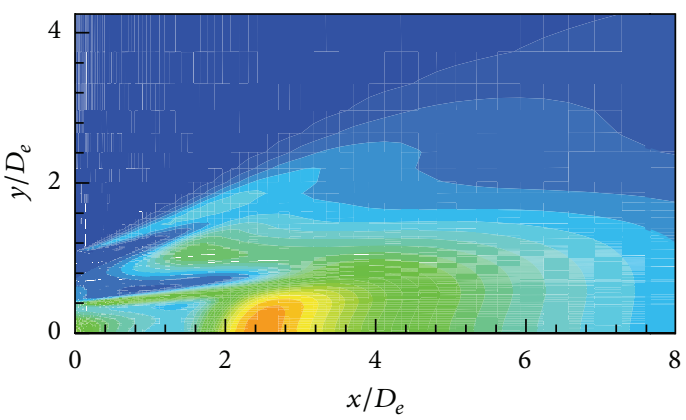

(d) SST $k-\omega$

FIGURE 12: Turbulent kinetic energy contours of wide side.

enable a faster transport of turbulent kinetic energy to RNG $k-\varepsilon$ model. The $k-\varepsilon$ T. C. model gives the fastest transport of turbulent kinetic energy. This could be more obviously observed in the turbulent kinetic energy contours shown in Figure 12 and Figure 13. The peak turbulent kinetic energy region produced in the jet shear layer by $k-\varepsilon$ T. C. model mostly appears before $x / D_{e}=2$, while for the other models the peak region mostly appears after $x / D_{e}=2$. It can also be seen that the diffusion of turbulent kinetic energy region in $z$ direction in narrow side predicted by $k-\varepsilon$ T. C. model is slightly quicker than the models with no temperature correction.
5.2. Temperature Distribution of the Jet. Figure 14(a) shows the computed temperature distribution along the centerline of the jet from the 2D nozzle. Similar improvement on temperature core region length prediction is observed as that of velocity core region prediction. The measured temperature core length is about 0.5 , while the simulated core lengths are $0.593,0.987,1.221$, and 1.125 for $k-\varepsilon$ T. C. model, Standard $k-\varepsilon$, RNG $k-\varepsilon$, and SST $k-\omega$ model, respectively. Although all of the four turbulence models give a longer core region prediction, the $k-\varepsilon$ T. C. model predicted a core region only 0.2 times longer than the measured value; it produces a much better result compared to the other three models which produce 

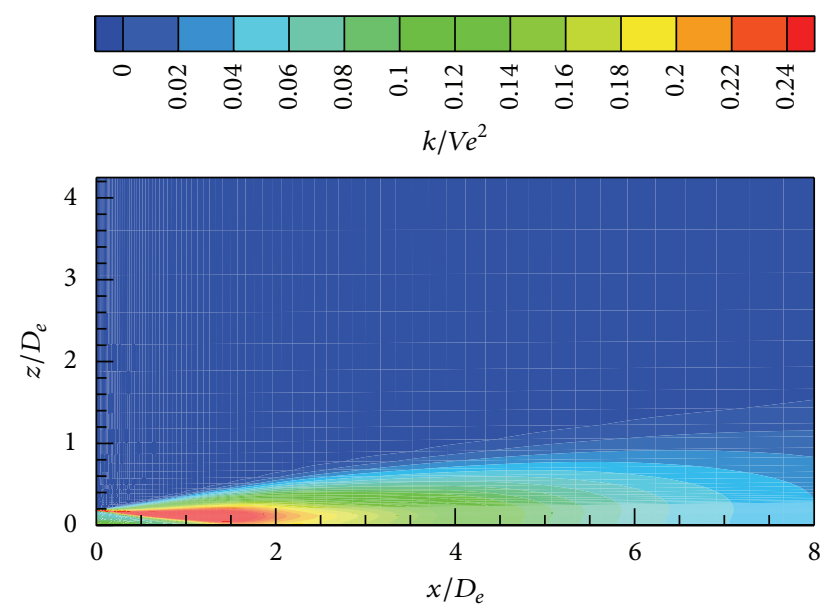

(a) $k-\varepsilon$ T. C.

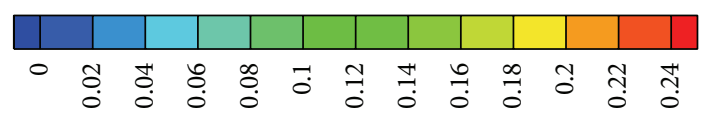

$k / V e^{2}$

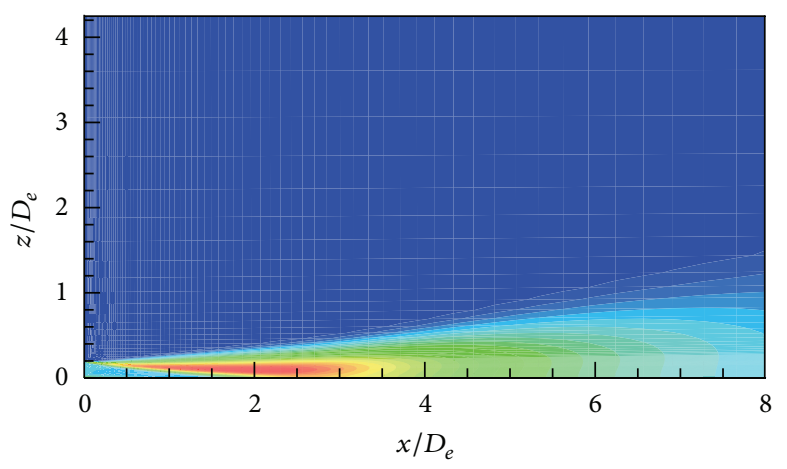

(c) RNG $k-\varepsilon$
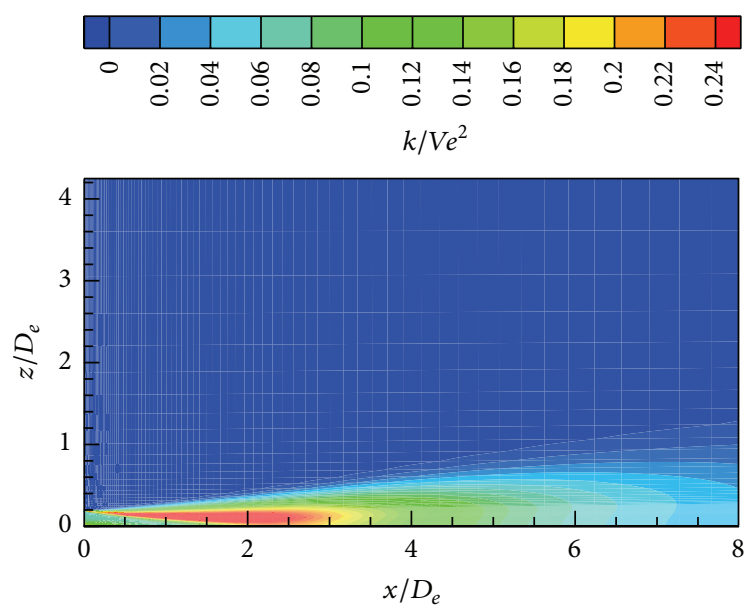

(b) Standard $k-\varepsilon$
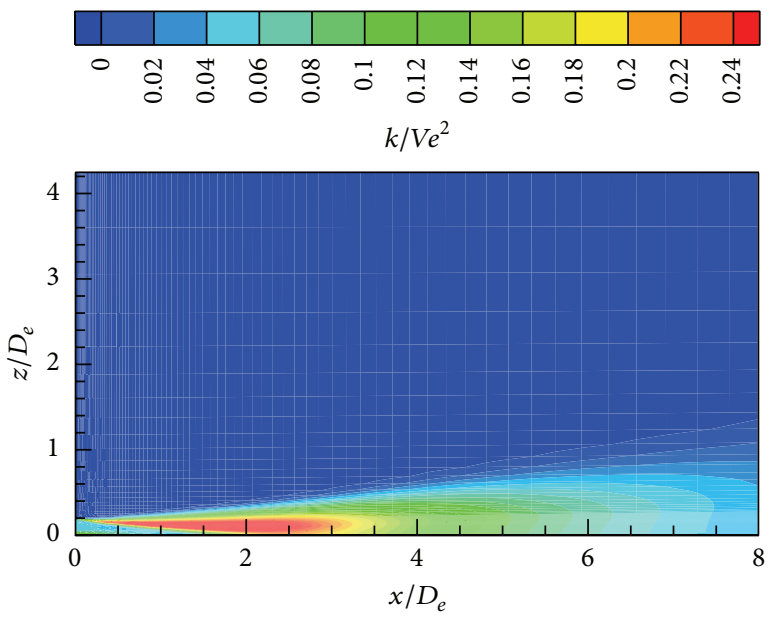

(d) SST $k-\omega$

FIGURE 13: Turbulent kinetic energy contours of narrow side.

a core region about 1 1.4 times longer than the measured value. The differences caused by different turbulence models are about $8.5 \%-106 \%$, and the errors between predictions and experiments are about $19 \%-144 \%$.

Figures 14(b) and 14(c) show the static temperature distribution of middle section from wide side and narrow side, respectively. In the core region, all the four turbulence models can give a reasonable result. But in the mixing region, the deviations are increased obviously, and the computational high temperature region is significantly larger than the experimental values. The computed temperature profiles by $k-\varepsilon$ T. C. model are more flat than other nontemperature turbulence models for the rapid rate of jet decay. And from Figure 14(b), it can be seen that the results of small $x / D_{e}$ are not very good when the $k-\varepsilon$ T. C. model is used. From the viewpoint of the In general, the computed temperature of the jet from $2 \mathrm{D}$ nozzle using $k-\varepsilon$ T. C. model is comparatively better than the other models.

5.3. Infrared Radiation of the Jet in $90^{\circ}$ to the Nozzle Axis. Figure 15 shows the comparison of computed and measured spectral radiation intensity $I_{\lambda}$ in the direction $90^{\circ}$ to the nozzle axis, and all the data are normalized. Only the maximum error bars are shown, since the small error bar will coincide with the symbols representing the experiment values. It can be observed that the spectral radiation intensity of the hot jet is mainly concentrated within a wavelength band of $4.15 \mu \mathrm{m} \sim 4.6 \mu \mathrm{m}$. The appearance of the double peaks is caused by the emission of the high temperature combustion production of $\mathrm{CO}_{2}$ in the jet and the absorption of the low temperature $\mathrm{CO}_{2}$ in atmosphere. With the use of the standard $k-\varepsilon$, RNG $k-\varepsilon$, and SST $k-\omega$ model, the predicted peak values of the spectral radiation are significantly higher than the measured data, and the predicted wavelength band widths of the spectrum of the radiation near the wavelength of $4.5 \mu \mathrm{m}$ are also obviously wider than the measured data. However, with the use of the $k-\varepsilon$ T. C. model, a significantly improved prediction is observed.

Table 3 gives the comparison of computed and measured integral intensity integrated from wavelength $4 \mu \mathrm{m} \sim 5 \mu \mathrm{m}$, where $I_{c}$ represents a calculated value and $I_{E}$ represents a measured value. It can be seen that, for the standard $k-\varepsilon$ 


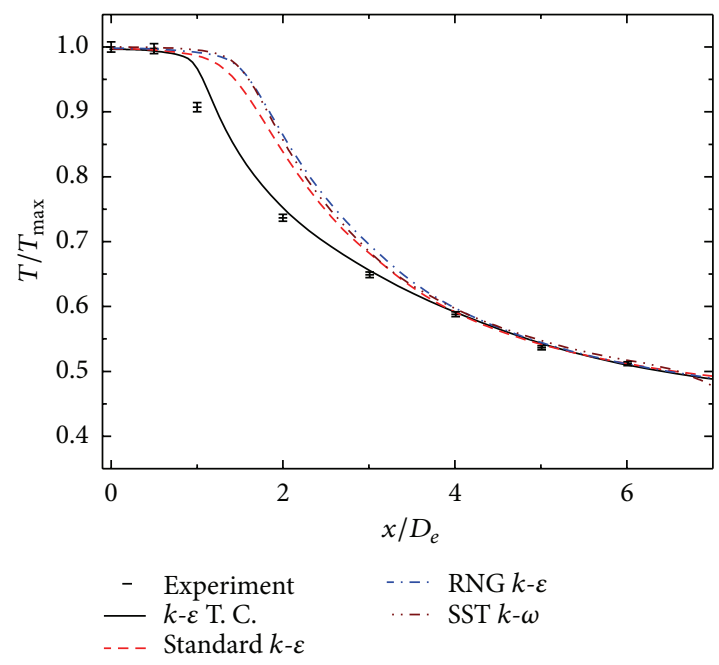

(a) Static temperature distribution along centerline of the nozzle

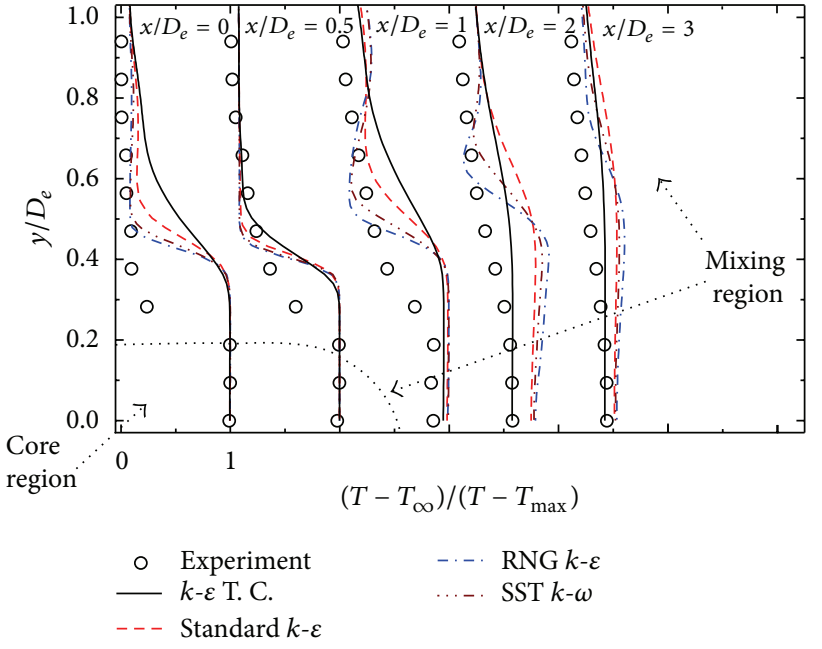

(b) Static temperature distribution (wide side)

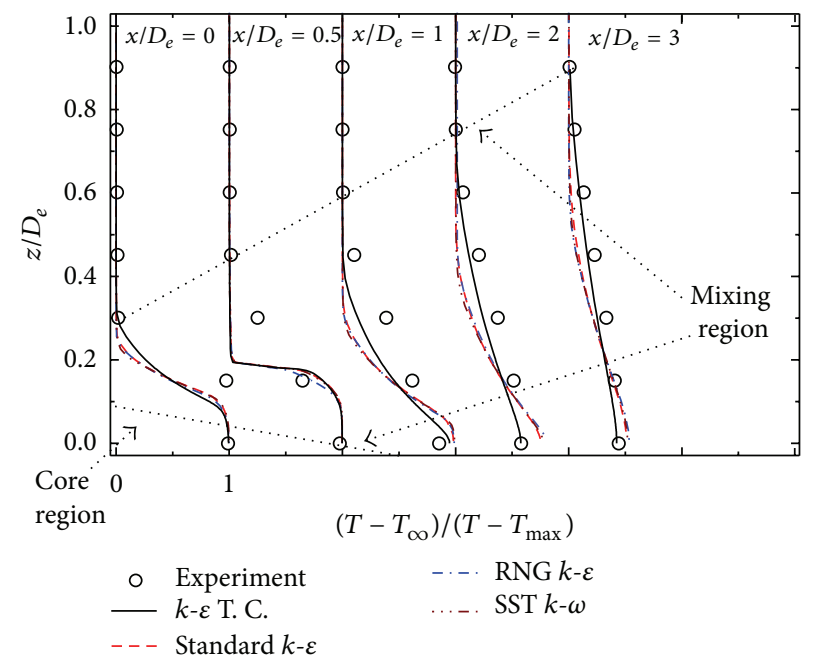

(c) Static temperature distribution (narrow side)

FIGURE 14: Computed temperature of the jet from the 2D nozzle compared with experimental data.

TABLE 3: Integral intensity error of the $2 \mathrm{D}$ nozzle $\left(4 \mu \mathrm{m} \sim 5 \mu \mathrm{m},\left(I_{C}-\right.\right.$ $\left.\left.I_{E}\right) / I_{E} \times 100 \%\right)$.

\begin{tabular}{lcccc}
\hline & Standard $k-\varepsilon$ & RNG $k-\varepsilon$ & SST $k-\omega$ & $k-\varepsilon$ T. C. \\
\hline Wide side & $70.7 \%$ & $86.5 \%$ & $74.4 \%$ & $26.0 \%$ \\
Narrow side & $27.9 \%$ & $42.5 \%$ & $33.0 \%$ & $-5.8 \%$ \\
\hline
\end{tabular}

model, RNG $k-\varepsilon$ model, and SST $k-\omega$ model, the error of prediction in wide and narrow side is larger than $70.7 \%$ and $27.9 \%$, respectively, while the $k-\varepsilon$ T. C. model reduces the error to $26.0 \%$ and $-5.8 \%$, respectively.

\section{Conclusion}

(1) The predicted lengths of the velocity and temperature core region by standard $k-\varepsilon$ model, RNG $k-\varepsilon$ model, and SST $k$ $\omega$ model are obviously longer than the measured data. For this reason, the computed infrared radiation intensities are significantly higher than the measured data. The maximum error is up to $80 \%$ or more. Because the numerical uncertainty and the experiment uncertainty are small, the errors between predictions and experiments are mainly caused by different turbulence models.

(2) The temperature corrected $k-\varepsilon$ T. C. model directly increases the turbulent viscosity, causing more rapid mixing. This model dramatically improves the computed velocity distribution and temperature distribution. In turn, the accuracy of the predicted infrared radiation is improved evidently.

(3) Although the $k-\varepsilon$ T. C. model gives a good prediction for the centerline velocity and temperature distribution of the $2 \mathrm{D}$ hot gas jet, it cannot make satisfactory prediction on the locations off the centerline due to the flow complexity. The computation error of the $2 \mathrm{D}$ hot jet infrared radiation using $k-\varepsilon$ T. C. model is the smallest but still up to $26 \%$. To further 


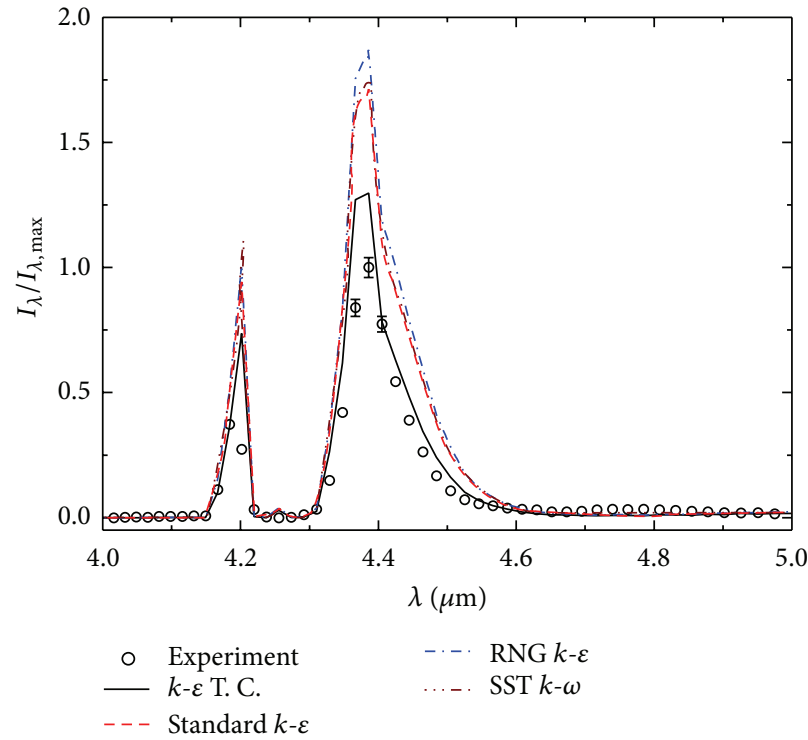

(a) Wide side

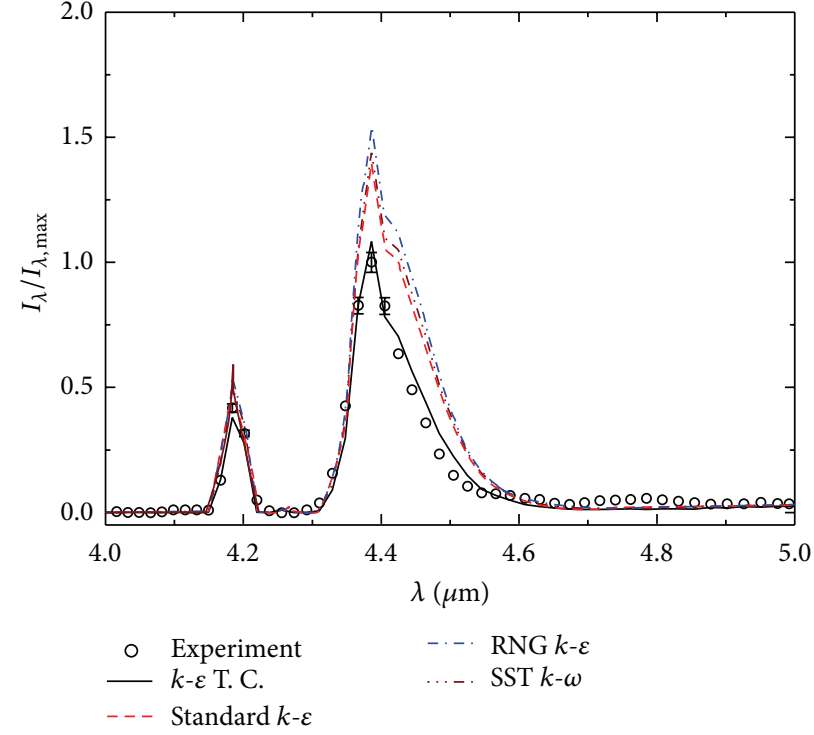

(b) Narrow side

FIGURE 15: Spectral infrared radiation of 2D nozzle.

improve the accuracy, a more accurate turbulence model is needed.

\section{Conflict of Interests}

The authors declare that there is no conflict of interests regarding the publication of this paper.

\section{References}

[1] C.-C. Liu, H.-H. Ji, W. Huang, and F.-F. Yang, "Numerical simulation on infrared radiation characteristics of serpentine 2D nozzle," Journal of Aerospace Power, vol. 28, no. 7, pp. 14821488, 2013 (Chinese).

[2] B. Wu, J.-Z. Zhang, Y. Shan, and W.-R. Shao, "Numerical research on infrared radiation characteristics of two-dimension nozzle with shelter covering," Journal of Aerospace Power, vol. 26, no. 10, pp. 2287-2292, 2011 (Chinese).

[3] M.-D. Luo, H.-H. Ji, W. Huang, X. Cai, B. Zhang, and C. Gao, "Experiment on spectral infrared radiation characteristics of exhaust jet from 2D nozzle of turbofan engine," Journal of Propulsion Technology, vol. 28, no. 2, pp. 152-156, 2007.

[4] C. Fureby, M. Henriksson, O. Parmhed, L. Sjökvist, and J. Tegnér, "CFD predictions of jet engine exhaust plumes," in Proceedings of the 38th AIAA Fluid Dynamics Conference and Exhibit, pp. 2008-3727, June 2008.

[5] W. P. Jones and B. E. Launder, "The prediction of laminarization with a two-equation model of turbulence," International Journal of Heat and Mass Transfer, vol. 15, no. 2, pp. 301-314, 1972.

[6] S. A. Orszag, V. Yakhot, W. S. Flannery et al., "Renormalization group modeling and turbulence simulations, near-wall turbulent flows," in Proceedings of the International Conference, pp. 1031-1046, 1993.

[7] F. R. Menter, "Two-equation eddy-viscosity turbulence models for engineering applications," AIAA Journal, vol. 32, no. 8, pp. 1598-1605, 1994.
[8] Y.-P. Hu, H.-H. Ji, and T.-T. Bai, "Effect of turbulence models on numerical simulation of flow and infrared radiation characteristic of aero-engine exhaust system," Journal of Aerospace Power, vol. 27, no. 11, pp. 2414-2420, 2012 (Chinese).

[9] M. A. Dembowski and N. J. Georgiadis, "An evaluation of parameters influencing jet mixing using the WIND navierstokes code," NASA/TM 2002-211727, 2002.

[10] J. M. Seiner, M. K. Ponton, B. J. Jansen et al., "The effects of temperature on supersonic jet noise emission," DGLR/AIAA 92-02-046, 1992.

[11] K. S. Abdol-Hamid, S. Paul Pao, S. J. Massey, and A. Elmiligui, "Temperature corrected turbulence model for high temperature jet flow," Journal of Fluids Engineering, vol. 126, no. 5, pp. 844850, 2004.

[12] C. K. W. Tam and A. Ganesan, "Modified $k-\varepsilon$ turbulence model for calculating hot jet mean flows and noise," AIAA Journal, vol. 42, no. 1, pp. 26-34, 2004.

[13] S. J. Massey, R. H. Thomas, K. S. Abdol-Hamid et al., "Computational and experimental flow field analyses of separate flow chevron nozzles and pylon interaction," AIAA 2003-3212, 2003.

[14] N. J. Georgiadis, D. A. Yoder, and W. A. Engblom, "Evaluation of modified two-equation turbulence models for jet flow predictions," AIAA Journal, vol. 44, no. 12, pp. 3107-3114, 2006.

[15] X. Li, Eriqitai, and Q. Wang, "Calculation of aircraft exhaust system infrared radiation using temperature corrected turbulence model," Procedia Engineering, vol. 32, pp. 23-28, 2012.

[16] X.-X. Li, "Numerical simulation of infrared radiation characteristic of exhaust system using Tam-Ganesan turbulence model," Journal of Aerospace Power, vol. 27, no. 10, pp. 2384-2389, 2012 (Chinese).

[17] M. Akbarzadeh, M. Birouk, and B. Sarh, "Numerical simulation of a turbulent free jet issuing from a rectangular nozzle," Computational Thermal Sciences, vol. 4, no. 1, pp. 1-22, 2012.

[18] M. Araki, Y. Ijuin, S. Nishida et al., "Mean-flow and acoustic characteristics of cold jets from a rectangular hypersonic 
nozzle," Journal of Propulsion and Power, vol. 30, no. 1, pp. 221232, 2014.

[19] J. R. Berg, S. J. Ormiston, and H. M. Soliman, "Prediction of the flow structure in a turbulent rectangular free jet," International Communications in Heat and Mass Transfer, vol. 33, no. 5, pp. 552-563, 2006.

[20] M. F. Modest, Radiative Heat Transfer, Adademic Press, 2th edition, 2003.

[21] ANSYS FLUENT User's Guide, ANSYS, Canonsburg, Pa, USA, 2011.

[22] F. Stern, R. V. Wilson, H. W. Coleman, and E. G. Paterson, "Comprehensive approach to verification and validation of CFD simulations-part 1: methodology and procedures," Journal of Fluids Engineering, vol. 123, no. 4, pp. 793-802, 2001.

[23] R. V. Wilson, F. Stern, H. W. Coleman, and E. G. Paterson, "Comprehensive approach to verification and validation of CFD simulations-part 2: application for rans simulation of a cargo/container ship," Journal of Fluids Engineering, vol. 123, no. 4, pp. 803-810, 2001. 


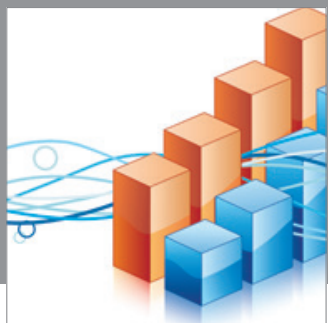

Advances in

Operations Research

mansans

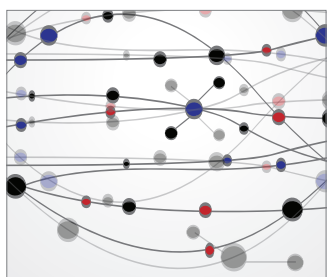

The Scientific World Journal
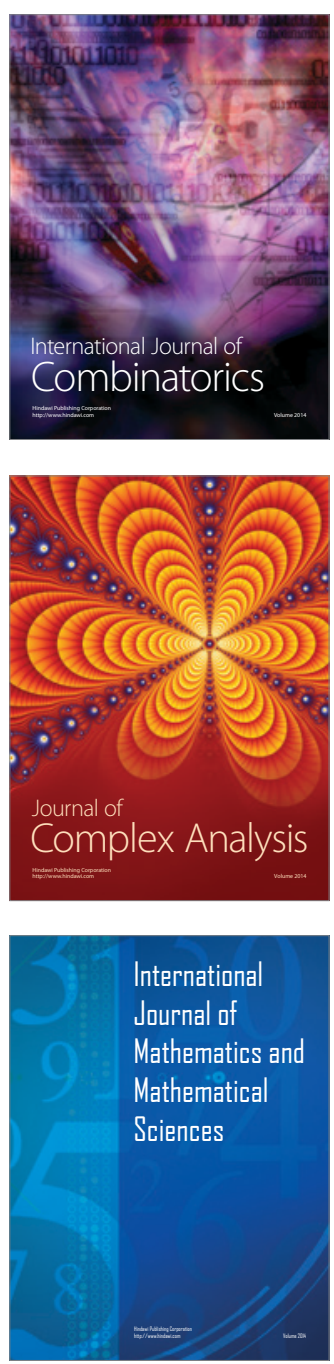
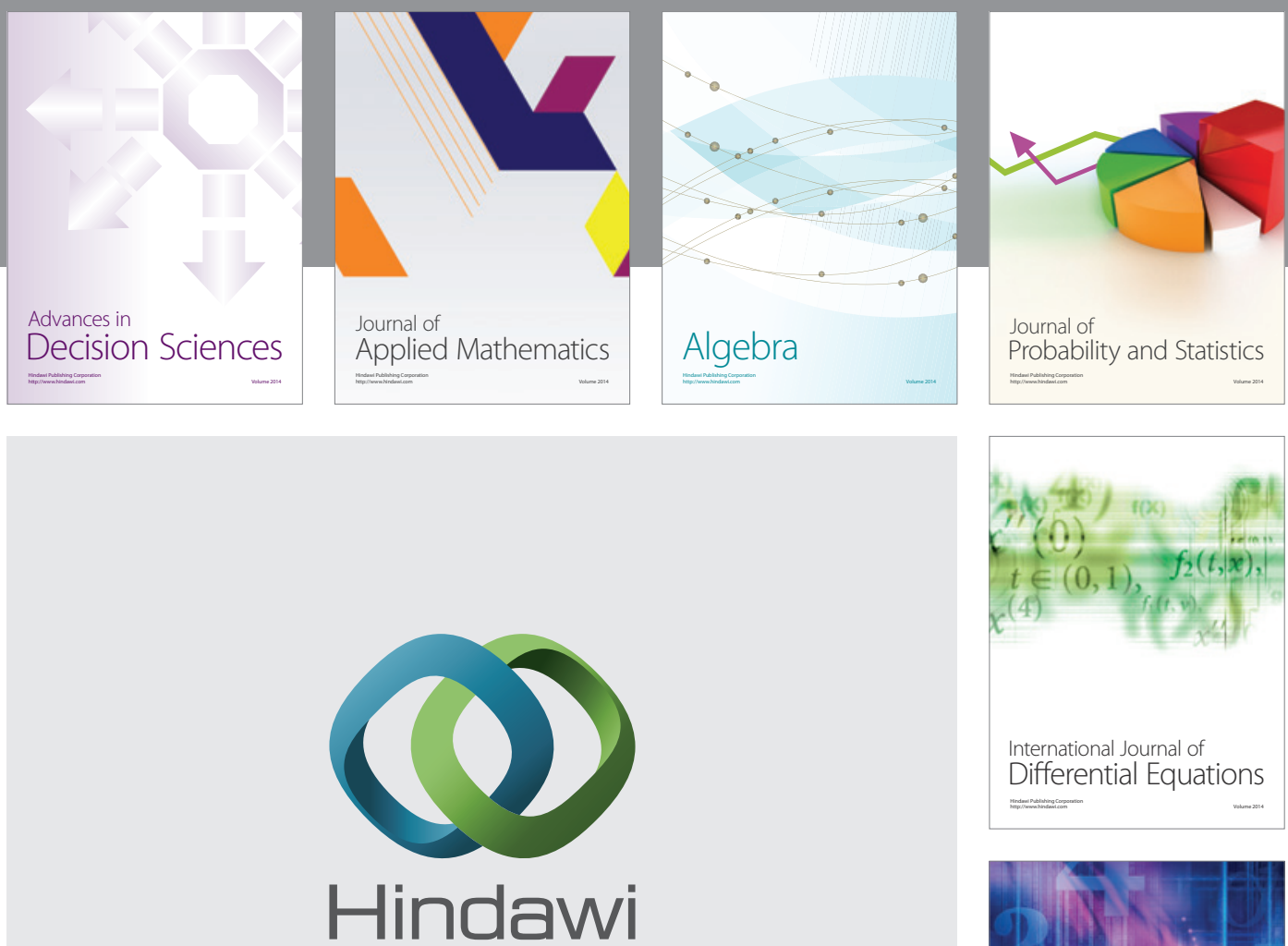

Submit your manuscripts at http://www.hindawi.com
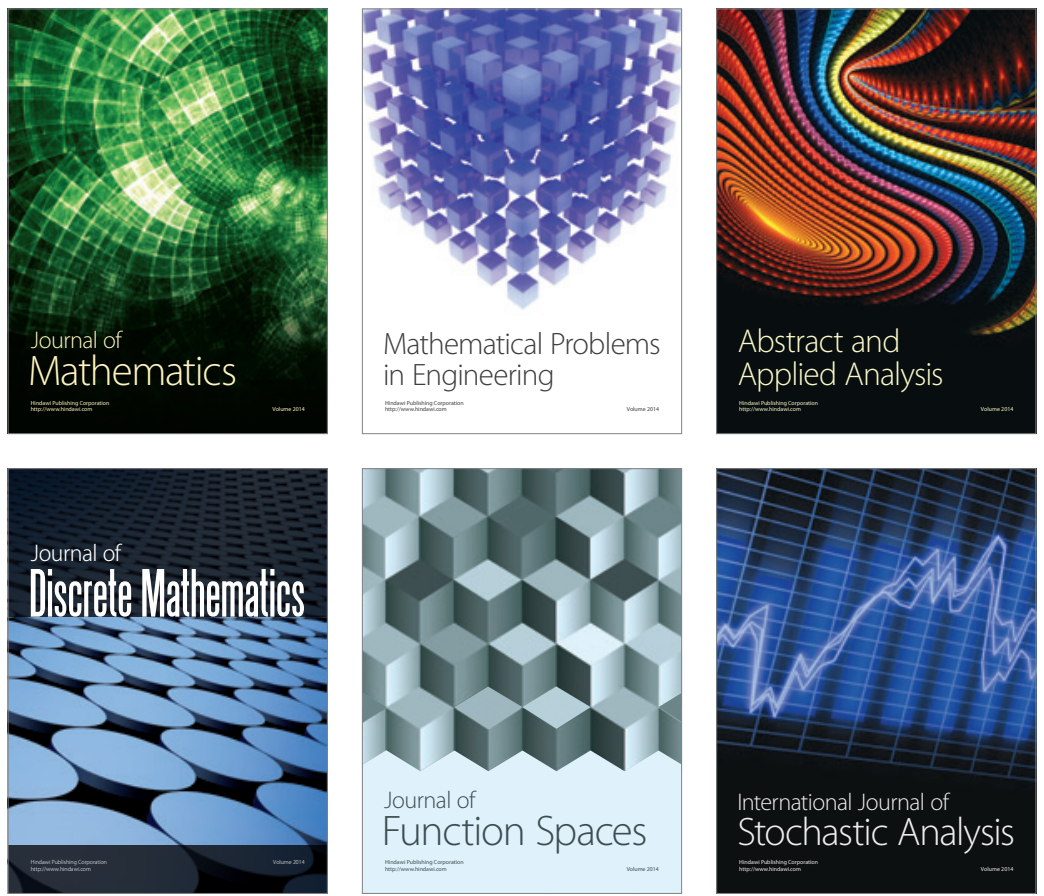

Journal of

Function Spaces

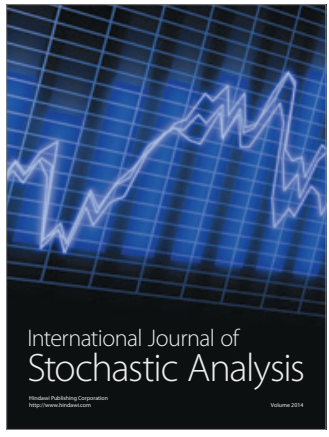

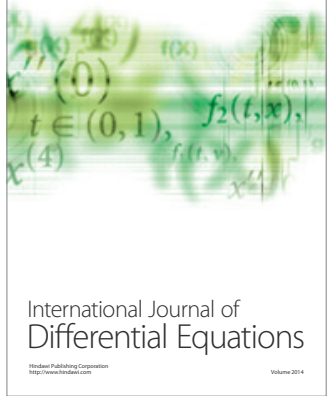
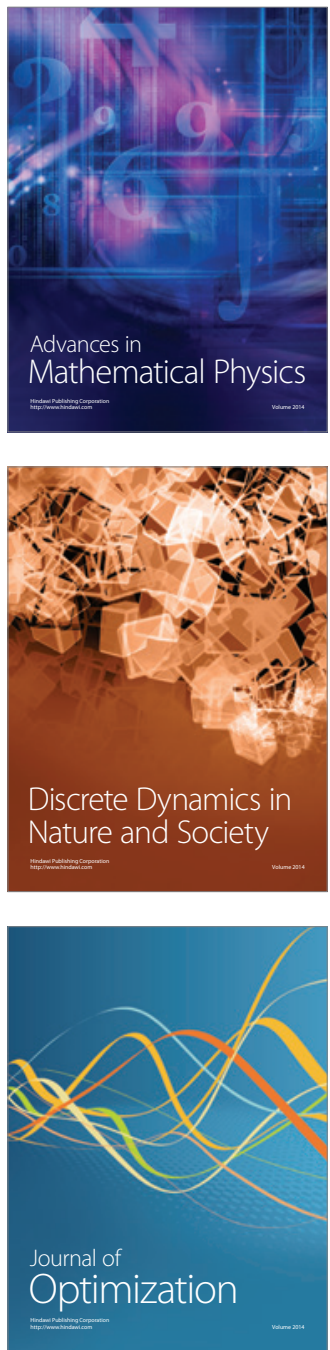